\title{
EL ESTUDIO DE CASOS COMO METODOLOGÍA DE INVESTIGACIÓN Y SU IMPORTANCIA EN LA DIRECCIÓN Y ADMINISTRACIÓN DE EMPRESAS
}

\author{
EDGAR CASTRO MONGE \\ Escuela Ciencias de la Administración \\ Universidad Estatal a Distancia, Costa Rica \\ edcastro@uned.ac.cr
}

\section{RESUMEN}

Son diversos los estudios realizados por diferentes autores que han señalado la gran importancia que tiene la investigación basada en estudios de casos en la Administración y Dirección de Empresas, ya que esta herramienta es una de las más utilizadas en la metodología de investigación cualitativa. El ensayo trata de dar las bases para justificar la importancia y el uso de esta metodología de investigación, y pretende a la vez realizar una comparación entre el estudio de casos (investigación cualitativa) y la investigación cuantitativa tratando de refutar algunos prejuicios y cuestionamientos que varios autores realizan al estudio de casos. Asimismo, se pretende dar las bases teóricas para desarrollar eficientemente un estudio de casos.

PALABRAS CLAVES: INVESTIGACIÓN CUALITATIVA, ESTUDIO DE CASOS, ADMINISTRACIÓN DE EMPRESAS

\section{ABSTRACT}

Studies by different authors have shown the importance of research based on case studies for Business Administration and Management. This is because this tool is commonly used as part of qualitative research methodologies. To provide the basis to justify the importance and use of this research methodology is intended, as well as a comparison of case studies (qualitative research) and quantitative research. Some prejudices and questioning made by some authors regarding case studies are refuted. Furthermore, the theoretical bases to efficiently conduct a case study are outlined.

KEYWORDS: QUALITATIVE RESEARCH, CASE STUDIES, BUSINESS ADMINISTRATION.

\section{INTRODUCCIÓN}

El estudio de casos, se ha convertido en un método de investigación importante en la Administración de empresas, aún cuando los investigadores de corte cuantitativo lo cuestionan alegando que sus conclusiones no son generalizables estadísticamente; sin embargo, la investigación cualitativa, específicamente con el estudio de casos, no representa a una muestra de una población o universo, son casos específicos los que se estudian buscando con esta metodología de investigación una generalización analítica y no estadística ampliando y generalizando teorías.

El uso del estudio de casos ofrece importantes resultados e información que no puede ser encontrada por medio de los métodos cuantitativos y que es muy valiosa para la toma de decisiones en las empresas. Son frecuentes las investigaciones que utilizan esta metodología que dan como resultado publicaciones en revistas de gran difusión y calidad en el área de la Dirección y Administración de empresas. 
Con el estudio de casos se pretende encontrar nuevas evidencias o situaciones de un fenómeno, la diferencia de lo que se está estudiando con su universo, la formulación de nuevas teoría de la realidad social, lo que se busca es encontrar las respuestas a preguntas en un escenario y momento dado, de ahí que no son formulaciones de verdades universales.

El estudio de casos como metodología de investigación, no debe ser confundido con el análisis o estudio de casos como herramienta pedagógica, que busca analizar una realidad o un aspecto en concreto y fomentar la discusión y el debate.

Se pretende en este ensayo, dejar claro que aun cuando los métodos cuantitativos y cualitativos pueden tener diferentes objetivos, uno no neutraliza al otro y que en el presente se ha dado una tendencia a la fusión y el uso de ambos métodos, ya que utilizan varias fases metodológicas similares.

De esta manera, se trata de lograr con las reflexiones planteadas, la importancia que tiene el estudio de casos en la actualidad, dando a conocer los aspectos relevantes y necesarios para poder realizar una investigación de este tipo correctamente y no dejar ningún aspecto importante de lado que le brinde a la investigación la validez, tanto interna como externa, para que el estudio refleje y explique verdaderamente el fenómeno, situación o aspecto estudiado.

\section{La investigación cualitativa}

A lo largo de la Historia de la Ciencia, ha existido diversidad de corrientes en el pensamiento, algunas de ellas están relacionadas con el Estructuralismo, el Materialismo Dialéctico, el Positivismo, el Empirismo, y la Fenomenología, las cuales han originado diferentes caminos en la búsqueda del conocimiento. Debido a diferentes premisas que las sustentan, estas corrientes se han polarizado en dos enfoques principales: el enfoque cuantitativo y el enfoque cualitativo de la investigación. Actualmente, ambos enfoques pueden formar parte de un mismo estudio o de una misma aplicación de dicho proceso, lo cual se denomina enfoque integrado multimodal. Desde hace algunos años se cree que ambos enfoques utilizados en conjunto enriquecen la investigación. No se excluyen ni se sustituyen, aunque hay quienes se muestran radicales ante alguna de estas posturas (Patton, 1990, Coser \& Hunt, 1994, Riege, 2003, Hernández, Fernández \& Baptista, 2003, Cepeda, 2006).

Los críticos del enfoque cuantitativo lo acusan de ser impersonal, frío, limitativo, cerrado y rígido. Por su parte, los detractores del enfoque cualitativo lo consideran vago, subjetivo, inválido, meramente especulativo, sin posibilidades de réplica y sin datos sólidos que apoyen las conclusiones (Hernández et al., 2003). En este mismo sentido, Stake (1995) sostiene que: "el objetivo de la investigación cualitativa es la comprensión, centrando la indagación en los hechos; mientras que la investigación cuantitativa fundamentará su búsqueda en las causas, persiguiendo el control y la explicación"(p. 37).

Para Dachler (1997), la investigación cualitativa y la cuantitativa tienen distintos objetivos y presuposiciones epistemológicas. La base del divorcio entre ambos enfoques se ha centrado en la idea de que un estudio con un enfoque puede neutralizar al otro. Sin embargo, revisando los estudios científicos en los últimos años se observa una tendencia creciente a la fusión de ambos métodos.

Grinnell (1997) señala que en términos generales, los dos enfoques -cuantitativo y cualitativoutilizan cuatro fases similares:

- Ambos enfoques logran probar y demostrar el grado en que las suposiciones o ideas tienen fundamento, a la vez revisan tales suposiciones o ideas sobre la base de las pruebas o del análisis. 
- Proponen nuevas observaciones para esclarecer, modificar o fundamentar las suposiciones; o incluso para generar otras.

- En los dos se da la observación y evaluación de fenómenos.

- Se establecen suposiciones como resultado de la observación y evaluación realizadas.

Es un hecho que ambos enfoques comparten esas etapas generales; sin embargo, cada uno tiene sus propias características. El enfoque cualitativo, por lo general, se utiliza en un primer orden para descubrir preguntas de investigación. En muchos casos, pero no necesariamente, se prueban hipótesis o proposiciones teóricas. Con frecuencia se basa en métodos de recolección de datos sin medición numérica, como las observaciones y las descripciones. En una gran mayoría de ocasiones, las preguntas e hipótesis surgen como parte del proceso de investigación y éste es flexible, y se mueve entre los eventos y su interpretación, entre las respuestas y el desarrollo de las teorías. El propósito de esta metodología consiste en reconstruir la realidad, tal y como la observan los autores de un sistema social que ha sido previamente definido. Por otra parte el enfoque cuantitativo, utiliza la recolección y el análisis de datos para contestar preguntas de investigación y probar hipótesis que han sido establecidas previamente, la mayor confianza la tiene en la medición numérica y frecuenta el uso de la estadística para establecer con exactitud patrones de comportamiento en una población (Stoecker, 1991, Miles \& Huberman, 1994, Grinnell, 1997).

Hernández et al. (2003) sostienen que ambos enfoques resultan muy valiosos y han realizado notables aportaciones al avance del conocimiento. Ninguno es intrínsecamente mejor que el otro y cada uno sirve a una función específica para conocer un fenómeno y para conducir a la solución de los diversos problemas y cuestionamientos.
La investigación cualitativa la define Orozco (1996) como un proceso de indagación de un objeto al cual el investigador accede a través de interpretaciones sucesivas, con la ayuda de instrumentos y técnicas, que le permiten involucrarse con el objeto para interpretarlo de la forma más integral posible. Quizás en esta definición se encierran las características más importantes de la perspectiva. En principio es un proceso, una construcción que en el tiempo se va superando, a la cual el investigador accede mediante interpretaciones sucesivas sobre el objeto indagado. Es decir, se conoce en progreso y no de una sola vez.

Strauss y Corbin (1990) argumentan que la investigación cualitativa es "cualquier tipo de investigación que produce resultados no encontrados por medio de procedimientos estadísticos u otros medios de cualificación" (p. 17). Por su parte, Mejía (2004) señala que la investigación cualitativa es el procedimiento metodológico que utiliza palabras, textos, discursos, dibujos, gráficos e imágenes para comprender la vida social por medio de significados y desde una perspectiva holística, pues se trata de entender el conjunto de cualidades interrelacionadas que caracterizan a un determinado fenómeno. Este tipo de investigación utiliza datos cualitativos, descripciones detalladas de hechos, citas directas, el habla de las personas y extractos de pasajes enteros de documentos para construir un conocimiento de la realidad social, en un proceso de conquista, construcción y comprobación teórica. La perspectiva cualitativa pretende encontrar lo distinto, lo propio, lo que diferencia aquello que se está explorando del conjunto que está integrando y eso es lo que se quiere intentar, para no reducir las palabras y los actos de las personas a presentaciones estadísticas, donde se pierde el centro del aspecto humano de la vida social (Taylor \& Bogdan, 1996). Los hallazgos cualitativos no son, por tanto, formulaciones de verdades: son la mejor respuesta que se puede 
obtener a una pregunta en un momento dado (Guba \& Lincoln, 1994).

El proceso de construcción de conocimiento siguiendo la metodología cualitativa es inductivo; es decir, que las etapas no son excluyentes sino que operan en un verdadero proceso de raciocinio, de lo particular a lo general, de manera interactiva, sin separar tajantemente la caracterización de la situación, el diseño metodológico, la recolección, la organización, el análisis y la interpretación de los datos, sino que la información se organiza y analiza en forma continua, con el fin de garantizar la representatividad y validez de los datos y orientar, a su vez, la búsqueda de nuevas evidencias que profundicen la comprensión del problema, que lo aclaren o lo caractericen con mayor precisión (Consuelo, 2005). Los métodos de investigación cualitativa son adecuados para la generación de la teoría, ya que el investigador construye o trata de completar el conocimiento (Wright, 1996).

Para Hernández et al. (2003), el principal desafío teórico supone asegurar la calidad de las investigaciones cualitativas. El desafío práctico está en determinar cuáles van a ser los resultados reales de la investigación, sobre todo cuando los investigadores:

a) No tienen ninguna experiencia en investigaciones cualitativas, ya sea teóricas o de aplicación práctica.

b) Deberían relacionar cuidadosamente enfoques de investigación con el objeto de la investigación, su contexto y los recursos disponibles, siendo preferible el no limitarse a un enfoque único.

c) Intentan demostrar la calidad, validez y viabilidad del enfoque particular utilizado en su investigación en foros poco familiarizados con dicho enfoque.
En los estudios cualitativos pueden desarrollarse preguntas o proposiciones antes, durante o después de la recolección y el análisis. Con frecuencia, estas actividades sirven, en primera instancia, para descubrir cuáles son las preguntas de investigación más importantes y, posteriormente, para refinarlas y responderlas o probar hipótesis. El proceso se mueve dinámicamente entre los hechos y su interpretación en ambos sentidos (Hernández et al., 2003).

En términos generales, los estudios cualitativos involucran la recolección de datos utilizando técnicas que no pretenden medir ni asociar las mediciones con números, tales como observación, revisión de documentos, discusión en grupo, evaluación de experiencias personales, entrevistas abiertas, inspección de historias, análisis semántico y de discursos cotidianos, interacción con grupos o comunidades e introspección. En este sentido, el análisis de datos es el paso más crítico en la investigación cualitativa (Miles \& Huberman, 1994). Es un proceso que consiste en consolidar, reducir e interpretar los datos que las personas aportaron y lo que el investigador ha visto o leído; esto significa dar sentido a los datos teniendo en cuenta las preguntas de investigación realizadas (Merriam, 1998).

Grinnell (1997) y Creswell (1997) describen las investigaciones cualitativas como estudios:

- Donde los significados se extraen de los datos y se presentan a otros, y no se necesita reducirlos a números ni tampoco deben analizarse de forma estadística.

- Que se conducen básicamente en ambientes naturales, donde los participantes se comportan como lo hacen en su vida cotidiana.

- Donde las preguntas de investigación no siempre se han conceptualizado, ni definido por completo. 
- Donde las variables no son definidas con el objetivo de manipularse ni de controlarse experimentalmente.

- En los que la recolección de datos es influida por las experiencias de los participantes en la investigación, más que por la aplicación de un instrumento de medición estandarizado, estructurado y predeterminado.

Newman (1991) por su parte, sintetiza las actividades principales del investigador cualitativo con las siguientes afirmaciones:

- El investigador es capaz de manejar paradoja, incertidumbre, dilemas éticos y ambigüedad.

- Produce datos en forma de notas extensas, diagramas y mapas, para generar descripciones bastante detalladas.

- Utiliza diversas técnicas de investigación y habilidades sociales de una manera flexible, de acuerdo con los requerimientos de la situación.

- El investigador está involucrado con las personas que se estudian y con sus experiencias personales y entienden a los miembros que son estudiados y desarrolla empatía hacia ellos.

- Observa los procesos sin irrumpir, alterar o imponer un punto de vista externo, sino tal y como son percibidos por los actores del sistema social.

- Mantiene una doble perspectiva: analiza los aspectos explícitos, conscientes y manifiestos, así como aquellos implícitos, inconscientes y subyacentes.

- Sigue una perspectiva holística, es decir, los fenómenos se conciben como un todo y no como partes.
- Adquiere un punto de vista interno -desde adentro del fenómeno-, aunque mantiene una perspectiva analítica o una distancia específica como observador externo.

- El investigador observa eventos ordinarios y actividades cotidianas tal y como suceden en sus ambientes naturales, además de cualquier acontecimiento inusual.

Las investigaciones cualitativas no pretenden generalizar de manera intrínseca los resultados a poblaciones más amplias, ni necesariamente obtener muestras representativas -bajo la ley de probabilidad-; incluso, no buscan que sus estudios lleguen a replicarse. Asimismo, se fundamentan más en un proceso inductivo -exploran y describen, luego generan perspectivas teóricas-.

\section{INVESTIGACIÓN CON ESTUDIO DE CASOS}

\section{El estudio de casos como metodología de investigación}

La investigación cualitativa ha ganado un amplio reconocimiento como enfoque válido y valioso. La metodología de investigación cualitativa es aplicable a una gran variedad de paradigmas de investigación -positivista, enfoque interpretativo y crítico-, dentro de los cuales hay muchos métodos de investigación como la investigación de la acción, los estudios de campo, etnografía y los estudios de casos. Todos estos métodos tienen un elemento común en la investigación cualitativa que consiste en recabar datos en forma de palabras e imágenes, que después serán analizadas mediante diversos métodos que no incluyen la estadística ni la cuantificación de ningún tipo (Morgan \& Smiricich; 1980, Hartley 1994; Gummesson, 2000; Carlson Engebretson \& Chamberlain, 2005; Cepeda, 2006; VanWynsberghe \& Khan, 2007). 
Son diversos los estudios que han señalado la gran importancia que tiene la investigación basada en estudio de casos, ya que esta herramienta es una de las más utilizadas en la metodología de investigación cualitativa (Barnes, 2001). Yin (1994) es uno de los principales autores en la investigación con estudio de casos, este autor es sin duda una referencia casi obligatoria para todos los que utilizan esta metodología de investigación. Yin señala que el estudio de casos es una investigación empírica que estudia un fenómeno contemporáneo dentro de su contexto de la vida real, especialmente cuando los límites entre el fenómeno y su contexto no son claramente evidentes. Una investigación de estudio de casos trata exitosamente con una situación técnicamente distintiva en la cual hay muchas más variables de interés que datos observacionales $y$, como resultado, se basa en múltiples fuentes de evidencia, con datos que deben converger en un estilo de triangulación; además, se beneficia del desarrollo previo de proposiciones teóricas que guían la recolección y el análisis de datos.

Bonache (1999), señala que se presentan algunos errores por la asociación de esta herramienta de investigación con el estudio de casos como herramienta pedagógica. La confusión del estudio de casos como herramienta de investigación con el estudio de casos como herramienta de enseñanza ha supuesto, en algunas ocasiones, descuidos y falta de sistematización y rigor por parte de los investigadores. Confirma este autor que, cuando el objetivo es la enseñanza, el material que constituye el caso puede ser deliberadamente alterado con el objetivo de ilustrar aspectos concretos y fomentar de esta manera la discusión y el debate, no presentando incluso una relación completa y exacta de los acontecimientos reales. Sin embargo, el objetivo de la utilización de esta estrategia en la investigación es precisamente presentar una relación completa y exacta de la realidad, donde no se puede, bajo ningún concepto, llevar a cabo la alteración de los datos, por lo que el investigador debe realizar un esfuerzo adicional para presentar fielmente toda la evidencia disponible.

Yin (1994) indica que uno de los principales prejuicios asociados a los estudios de casos es que sus conclusiones no son generalizables estadísticamente. Sin embargo, es claro que los estudios de casos no representan a una muestra de una población o de un universo concreto, por lo que no pueden ser generalizables estadísticamente, sino a proposiciones teóricas, ya que el objetivo del investigador es ampliar y generalizar teorías - generalización analítica- y no enumerar frecuencias -generalización estadística-. Es así como el propósito de esta herramienta de investigación es comprender la interacción entre las distintas partes de un sistema y de las características importantes de éste, de forma tal que el análisis realizado pueda ser aplicado de manera genérica (Hartley, 1994), incluso a partir de un caso único, en cuanto que se logra una comprensión los procesos, de la estructura y las fuerzas impulsoras, más que un establecimiento de correlaciones o relaciones de causa y efecto (Gummesson, 2000).

Existe la idea de que para llevar a cabo un estudio de casos se requiere demasiado tiempo, y tienen como resultado documentos muy largos y difíciles de leer. Un estudio de casos requiere de una gran dedicación; sin embargo, la redacción de éstos no tienen que ser largas narraciones, ni este tipo de estudios necesariamente debe llevar un excesivo tiempo, pues siempre los datos deberán obtenerse mediante observación directa o métodos etnográficos (Sosa, 2003).

Una de las discusiones más interesantes con respecto a los casos, es lo que señalan los autores Otley y Berry (1994), quienes afirman que el estudio de casos es un método que puede ser usado por distintas metodologías. Pero para Hartley (1994), el estudio de casos no es un método. En cambio, para Silverman (1993) y para VanWynsberghe y Khan (2007), el estudio de casos es una 
metodología que utiliza varios métodos, ya que entiende por metodología un enfoque general para estudiar un problema de investigación, mientras que al método lo define como una técnica específica para recoger datos.

Es importante hacer énfasis en que la metodología del estudio de casos no es sinónimo de investigación cualitativa, ya que los estudios de casos pueden basarse en cualquier combinación de evidencias cuantitativas y cualitativas (Ghauri et al., 1995); incluso, pueden fundamentarse sólo en evidencias cuantitativas y no tienen que incluir siempre observaciones directas y detalladas como fuente de información. Por su parte, Larsson (1993) señala que nada impide que se apliquen técnicas estadísticas u otros métodos cuantitativos, como cuando se quiere descubrir ciertos patrones en los datos existentes. Bryman (1984), aceptando los principios de fiabilidad y validez de la investigación, considera que los casos no se oponen sino que complementan la metodología cuantitativa. Es así cómo los estudios de casos pueden incluir planes comerciales, análisis de documentos, entrevistas, cuestionarios, datos financieros, memorandos, encuestas y observaciones de las acciones e interacciones de directivos y de empleados, integrando información con el objetivo de comprender, en la medida de lo posible, el fenómeno que se está sometiendo a estudio (Meredith, 1998; Eisenhardt, 1989).

En este mismo sentido, Hartley (1994) da a conocer que un estudio de casos no será definido siempre por las técnicas utilizadas sino por su orientación teórica y el énfasis en la comprensión de procesos dentro de sus contextos. No obstante, sí es cierto que la complejidad del estudio de los fenómenos sociales requiere de diferentes planteamientos y métodos específicos para su análisis y es más frecuente que éstos se centren, preferentemente, en sus características cualitativas, especialmente cuando el propósito es comprender e interpretar los sucesos en su globalidad (Stake, 1995). Para Smith y Zeithaml
(1999) el método de estudio de casos se ajusta muy bien a los objetivos de generar nuevos elementos en la investigación que se realiza y es un método recomendado porque habilita al investigador a ser sensible al carácter longitudinal del caso, a estudiarlo como un proceso de desarrollo a través del tiempo.

Algunos autores consideran que los estudios de casos son una forma de investigación que no puede utilizarse para contrastar proposiciones. Se debe defender, por un lado, que los estudios de casos, pueden servir para objetivos exploratorios, descriptivos y explicativos, y que pueden contribuir positivamente a la construcción y desarrollo de perspectivas teóricas rigurosas en torno a las organizaciones (Bonache, 1999).

\section{Clasificación de los estudios de casos}

Con una investigación de estudio de caso se pueden lograr diferentes objetivos: hacer una descripción, ofrecer explicaciones o interpretaciones sobre el fenómeno investigado, explorar sus características y funcionamiento o hacer una evaluación (Merriam, 1988; Yin 1994). La formulación de las soluciones provisionales y su papel cambiará en función de estos objetivos.

Muchas preguntas de tipo "¿qué?" son exploratorias o descriptivas y se contestan realizando encuestas o consultando bases de datos, ya que lo que se pretende es describir la incidencia o la prevalencia de un fenómeno, o bien hacer predicciones acerca de ciertos resultados. Las preguntas "¿cómo?" y “ipor qué?" son más explicativas y son especialmente relevantes porque sus respuestas son las teorías, ya que tratan vínculos operativos cuya evolución debe seguirse a lo largo del tiempo, y no simplemente frecuencias o incidencias (Yin, 1994; Yacuzzi, 2005).

Es así cómo Yin (1994) señala que existen diversas clasificaciones de los estudios de casos, destacando, entre ellas, la que se realiza en función 
de los objetivos del estudio y la que se fundamenta en el número de casos objeto de análisis.

1) Atendiendo al objetivo de la estrategia de investigación. Se puede resumir la clasificación de estudios de casos propuesta por Yin (1994) en:

- Descriptivos, cuyo objetivo es analizar cómo ocurre un fenómeno organizativo dentro de su contexto real.

- Exploratorios, que buscan familiarizarse con un fenómeno o una situación sobre la que no existe un marco teórico bien definido.

- Ilustrativos, que de una u otra manera, ponen de manifiesto las prácticas de gestión de las empresas más competitivas.

- Explicativos, que tratan de desarrollar o depurar teorías, por lo que revelan las causas y los procesos de un determinado fenómeno organizativo.

2) Desde el punto de vista que se fundamenta en el número de casos objeto de análisis, se presenta la siguiente clasificación:

- Un único caso. Esta metodología es adecuada cuando el caso sea especial y tenga todas las condiciones necesarias para confirmar, desafiar o ampliar una determinada teoría. En una línea similar, Pettigrew (1990) indica que un único caso puede ser adecuado si el tratamiento del material de éste es suficientemente genérico o si la calidad y naturaleza de las conclusiones son únicas o fuertes.

- Múltiples o comparativos casos. En este tipo de estudio se hacen las mismas preguntas a los distintos casos, pero realizando una comparación de las respuestas para llegar a conclusiones importantes. De esta manera, las evidencias basadas en varios casos pueden ser consideradas más sólidas y convincentes, porque la intención en el estudio de casos múltiples es que coincidan los resultados de los distintos casos, y por supuesto esto permitiría añadir validez a la teoría propuesta. De hecho, cada caso debe tener un propósito determinado, de ahí que la elección de estos no se realiza según los criterios muestrales estadísticos sino por razones teóricas, buscando el conjunto de casos que sea representativo del fenómeno que se estará analizando. Según Langley y Royer (2006), en el estudio de casos múltiples, primero la lógica implica dibujar semejanzas o diferencias entre los casos a estudiar y cada caso puede ser al menos parcialmente descrito, porque no necesariamente todos los casos dentro de un colectivo deben ser conducidos exactamente del mismo modo, algunos casos pueden incluir objetivos específicos y conducidos con menos intensidad que otros.

Si se busca una relación con la cantidad de casos a estudiar, existe cierta controversia, Chiva (2001) establece que un estudio de casos múltiple requeriría un mínimo de cuatro unidades de análisis, aunque Eisenhardt (1989) sugiere que entre 4 y 10 casos es un número adecuado, y sostiene que cuanto mayor sea este número de unidades a estudiar, se puede alcanzar una mayor fiabilidad. Dyer y Wilkins (1991) sostienen que dos podrían ser ya suficiente número, y Voss Tsikriktsis, y Frohlich (2002), por su parte, defienden que no existe un número óptimo, sino más bien que los casos elegidos deben abarcar el conjunto de situaciones. Desde el punto de vista práctico, Yan y Gray (1994) afirman que la certeza se puede obtener después de un número pequeño de casos, pero, ¿cuantos casos? Se cree que esto es una cuestión de discrecionalidad. En general, según Mintzberg (1979), se consigue una mayor 
certidumbre cuanto mayor sea el número de casos, pero si se cree haber aislado las condiciones suficientes y necesarias para explicar y predecir el fenómeno estudiado, se podrá dar por concluida la investigación. Por su parte, Eisenhardt (1991) argumenta que el número de casos apropiado depende del conocimiento existente, del tema y de la información que se pueda obtener a través de la incorporación de estudios de casos adicionales. De acuerdo con lo anterior, Perry (1998) indica que no hay una guía precisa acerca del número de casos que deben ser incluidos, por lo que la decisión se deja al investigador. De manera similar, Eisenhardt (1989) recomienda que los casos deben adicionarse hasta que la saturación teórica de la muestra sea enriquecida.

Por otra parte, Cepeda (2006) describe dos tipos de casos que se clasifican en función del paradigma en el que el investigador se sitúa. De este modo se tiene, por un lado, los estudios de casos con un enfoque positivista y, por otro, los estudios de casos con un enfoque interpretativo.

Los estudios de casos con enfoque positivista presentan varias características importantes:

- No son aceptadas manipulaciones experimentales.

- No necesariamente a priori, se deben especificar las variables dependientes e independientes.

- Los resultados que se obtengan dependen básicamente de la capacidad de integración del investigador.

- El desarrollo de nuevas hipótesis puede dar como resultado cambios en la elección del lugar y en los métodos de recolección de datos.

- Se analizan una o pocas entidades -personas, grupos u organizaciones-.
- La complejidad de la unidad estudiada se analiza intensivamente.

- El estudio de casos se diseña más para las fases de exploración, clasificación y desarrollo de hipótesis o proposiciones en el proceso de generación de conocimiento.

- Este tipo de estudio se centra en situaciones actuales.

- El fenómeno es estudiado en su estado natural.

- Existen diversas fuentes y formas de obtener los datos.

- El estudio de casos es muy útil en el estudio de "¿por qué?" y ¿cómo?", ya que se refieren a las relaciones operativas que se establecerán a lo largo del tiempo más que en frecuencia e incidencia de éstas.

Por su parte, los estudios de casos con enfoque interpretativo presentan varios principios básicos:

- Principio de abstracción y generalización: requiere relacionar los detalles ideográficos revelados a partir de la interpretación de los datos mediante la aplicación de los principios de círculo hermenéutico y contextualización a la teoría, con los conceptos generales que describen la naturaleza de la comprensión humana y la acción social:

- Principio del razonamiento dialéctico: exige sensibilidad con las posibles contradicciones entre las preconcepciones teóricas que orientaron el diseño de la investigación y los resultados finales -la historia que los datos cuentancon sus subsecuentes ciclos de revisión.

- Principio de múltiples interpretaciones: requiere sensibilidad con las posibles diferencias de interpretaciones entre los participantes cuando se expresan en distintas narraciones o historias de la misma secuencia de hechos objeto de estudio 
- Principio de sospecha: necesita de la sensibilidad con los posibles sesgos y las distorsiones sistemáticas en las declaraciones obtenidas de los participantes.

- Principio fundamental del círculo hermenéutico: recomienda que toda la comprensión humana se alcanza por la interacción entre la comprensión de las partes interdependientes y el todo que forman.

- Principio de contextualización: exige una reflexión crítica del bagaje histórico y social del estado de la investigación, de esta forma la audiencia a la que va dirigido el caso pueda ver cómo se llega a la situación objeto de estudio.

- Principio de interacción entre los investigadores y los sujetos: exige una reflexión crítica sobre cómo los datos que recaba el investigador se construyen socialmente por la interacción entre los investigadores y los participantes.

En la tabla 1 se presenta un resumen de las diferentes clasificaciones de los estudios de casos.

\section{TABLA 1 \\ RESUMEN DE CLASIFICACIÓN DE LOS ESTUDIOS DE CASOS}

\begin{tabular}{|c|c|}
\hline CONCEPTO & CLASIFICACIÓN \\
\hline \multirow{4}{*}{$\begin{array}{l}\text { Según el objetivo de la estra- } \\
\text { tegia de investigación }\end{array}$} & Descriptivos \\
\hline & Exploratorios \\
\hline & Ilustrativos \\
\hline & Explicativos \\
\hline \multirow{2}{*}{$\begin{array}{l}\text { Con respecto al número de } \\
\text { casos que conforman un } \\
\text { estudio }\end{array}$} & Un único caso \\
\hline & $\begin{array}{l}\text { Múltiples o comparativos } \\
\text { casos }\end{array}$ \\
\hline \multirow{2}{*}{$\begin{array}{l}\text { En función del paradigma en } \\
\text { el que el investigador se sitúa }\end{array}$} & \\
\hline & Con enfoque interpretativo \\
\hline
\end{tabular}

Fuente: Elaboración propia a partir de Cepeda, 2006; Yin, 1994.

\section{El estudio de casos en administración y dirección de empresas}

En el presente existe gran interés en el uso de la investigación cualitativa en las diferentes áreas de la Administración de Empresas. Una de las justificaciones para este hecho está en la insatisfacción que se tiene con la información que brindan las investigaciones de corte cuantitativo (Weber, 2004). Las fuentes de esta insatisfacción son muy variadas: las restricciones existentes en las distribuciones de datos inherente al uso de estos métodos, la complejidad de los métodos multivariantes, los grandes tamaños muestrales que estos métodos exigen y las grandes dificultades que se presentan a la hora de comprender e interpretar los resultados de los estudios con métodos cuantitativos (Cepeda, 2006).

Los estudios de casos cada vez más se consideran como una valiosa herramienta de investigación (Gummesson, 2000). Hay algunas causas que dan sustento a este cambio fundamental de tendencia.

Para estudiar la creciente complejidad de los fenómenos organizativos, se necesita una investigación de carácter exploratorio y comprensivo más que la búsqueda de explicaciones causales. Para ello el estudio de casos es un método de investigación muy apropiado. En relación a esto, Mintzberg (1990) indica que no importa cuál sea el estado del campo, si es nuevo o maduro, toda investigación interesante explora. De hecho, cuando se realiza un estudio en profundidad en el campo de las organizaciones más complejas, se descubre que es necesario utilizar metodologías de investigación exploratoria en oposición a las consideradas rigurosas (VanWynsbergghe \& Khan, 2007).

Este cambio de tendencia también tiene sus bases en la mayor frecuencia de investigaciones y publicaciones de trabajos que utilizan esta importante metodología, principalmente en las 
revistas que tienen gran impacto, calidad y difusión en el área de la Organización y Administración de Empresas, lo que por supuesto brinda un mayor nivel de apoyo, legitimidad y credibilidad (Bonache, 1999, Carlson et al., 2005, Cepeda, 2006, VanWynsberghe \& Khan, 2007, Gibbert et al., 2008).

Stoecker (1991) señala que el estudio de casos en el ámbito empresarial tuvo su origen a principios del siglo pasado en las escuelas de negocios de Estados Unidos, donde su principal líder fue la Universidad de Harvard, que lo utilizó como metodología docente y de investigación de los fenómenos empresariales; sin embargo fue la Universidad de Chicago la que lideró en el mundo el empleo de esta metodología de investigación (Hamel et al., 1992). Posteriormente, se dieron varios años de estancamiento, pero fue necesario su uso justificando que los métodos cuantitativos presentan limitaciones para el estudio de realidades complejas y cambiantes como la empresarial, se generó así un resurgimiento en los años sesenta que resultó en un debate a favor de los métodos cualitativos. Es a partir de los años ochenta que surgen importantes contribuciones para la investigación con estudio de casos en el ámbito empresarial (Yin, 1989; Eisenhardt, 1989; Patton, 1990; Stoeker, 1991; Hamel, 1992, 1993; Stake, 1994; Rialp, 1998; Bonache, 1999; Fong, 2002; Carlson et al., 2005; Cepeda, 2006; VanWynsberghe \& Khan, 2007; Gibbert et al., 2008; entre otros). Estos aportes le dan un gran impulso a la metodología de estudio de casos situándola como una metodología importante dentro de los métodos científicos de uso generalizado.

Es así cómo, la búsqueda constante de nuevos y mejores modelos descriptivos y explicativos de los comportamientos de los mercados y las empresas requiere de metodologías que puedan, a partir del contacto con el objeto de estudio, generar nuevas explicaciones requeridas. Por ello, dadas las limitaciones de las aproximaciones cuantitativas para construir nuevas teorías, el estudio de casos resulta una metodología particularmente útil en la investigación relacionada con las empresas y con los mercados (Fong, 2002).

En este mismo sentido se puede agregar que los casos de investigación adoptan, en general, una perspectiva integradora, razón por la que el estudio de casos es una metodología de investigación muy utilizada en el análisis de las organizaciones por las distintas disciplinas científicas. Como prueba de ello, Grunow (1995), en su análisis sobre las estrategias de investigación en organización de empresas, analizó 204 estudios empíricos y la metodología del caso fue adoptada en más del 50 por ciento. Aunque todavía muchos académicos creen que los casos nos alejan de la manera normal en que se debe hacer ciencia, ya que ésta se tiende a identificar con los análisis estadísticos y las grandes muestras (Bonache, 1999).

Existe una larga presencia e influencia del estudio de casos en la literatura de la organización la cual se refleja en algunos de los trabajos empíricos más citados en los textos y manuales, entre los que destacan los de Mayo (1946), Lawrence y Lorsch (1967), Mintzberg (1979) y Hamel y Prahalad (1995). Hay otros estudios relacionados con los procesos de cambio estratégico en las empresas que operan en sectores maduros e introducen innovaciones organizativas (Stopford \& Baden-Fuller, 1994; Volberda, 1997). Por su parte, Dess y Davis (1984) y Robinson y Pearce (1988) estudiaron las estrategias competitivas de varias empresas, y otros estudios de casos analizan a las organizaciones que abandonan su negocio tradicional a favor de un nuevo negocio estratégico, aprovechando las capacidades existentes (Burgelman, 1994). Asimismo, hay estudios de empresas en las que las condiciones del entorno han influido notablemente en la trayectoria que seguían permitiéndose entrar en nuevos modelos de negocios (Volberda, 1997; Vouzas \& Kufidu, 2003). De esta manera, se pueden re- 
tomar las consideraciones de Mintzberg (1979) que hacía respecto a la investigación científica, abogando más por un planteamiento inductivo que deductivo.

En los últimos años el uso de la metodología del estudio de casos en la investigación relacionada con la empresa y también con las estrategias empresariales, ha visto un renacimiento y se ha incrementado considerablemente (Eisenhardt y Graebner, 2007; Siggelkow, 2007; Weick, 2007; Gibbert et al., 2008). El estudio de casos se ha erigido en los últimos tiempos como una de las metodologías de investigación científica con creciente utilización en las diversas áreas de la Economía de la Empresa. La creciente aceptación del estudio de casos en la investigación relacionada con la empresa, ha sido impulsada por una mejor comprensión de los criterios de calidad que pueden ser utilizados para evaluar una investigación mediante su uso. Estos criterios de valoración han alcanzado un considerable nivel de consenso y de difusión, como resultado de la publicación y presentación en eventos especializados de los trabajos asociados al debate sobre la importancia del uso de esta técnica de investigación (Eisenhardt, 1989; Stoecker, 1991; Villareal \& Landeta, 2007; Gibbert et al., 2008). De esta manera, el estudio de casos como metodología aplicada de investigación científica en el área de la administración de empresas está siendo cada vez más aceptado, sobre todo al comprobarse que el acceso a información de primera mano sirve para la toma de decisiones y es fundamental, ya que el cambio en las organizaciones requiere de un tipo de análisis no realizable con la suficiente profundidad a través del estudio de un número elevado de observaciones (Rialp, 1998).

En este mismo sentido, Sarabia (1999) indica que, en lo metodológico, la investigación científica actual es una espiral inductivo-hipotéticodeductivo con dos pasos procesales esenciales:
- Fase heurística o de descubrimiento: fase hecha de observación, descripción, reflexión y generalización inductiva, con miras a generar hipótesis (lo que podría ser verdadero como solución al problema, respuesta a la cuestión o explicación del fenómeno).

- Fase de justificación-confirmación: proceso de comprobación del fundamento de una hipótesis por medio de un procedimiento o dispositivo previsto al efecto (y susceptible de ser reproducido).

De acuerdo con lo anterior, este autor considera que las metodologías útiles para la fase de descubrimiento son las cualitativas. De allí que la aplicación de la metodología cualitativa en el mundo empresarial sea cada vez mayor, debido a la permanente necesidad del tipo de información obtenida a través de la misma, tanto en el ámbito de la dirección y organización como en el ámbito comercial, estratégico o de marketing.

Es necesario hacer énfasis en que el estudio de casos es una investigación en profundidad para analizar los procesos implicados en el fenómeno objeto de estudio, en los que el fenómeno no se aísla de su contexto. De hecho, los estudios de casos, como ejemplos reales de la experiencia de las empresas, son capaces de mostrar sus propias historias sobre el desarrollo del tema a estudiar en la práctica y de cómo el contenido, el contexto y las políticas de las empresas interaccionan en la dinámica de la empresa (Dawson, 1997).

Es así cómo se puede resumir que el estudio de casos es uno de los métodos más apropiados para aprender la realidad de una situación, en los que se requiere explicar relaciones causales complejas, realizar descripciones de perfil detallado, generar teorías o aceptar posturas teóricas exploratorias o explicativas, analizar procesos de cambio longitudinales y estudiar un fenómeno que sea, esencialmente, ambiguo, complejo e incierto (Villareal y Landeta, 2007). 
En este mismo sentido, el uso de la estrategia del estudio de casos como metodología de investigación presenta grandes posibilidades en la explicación de fenómenos contemporáneos ubicados en su entorno real (Yin, 1989; Eisenhardt, 1989). La transformación de los mercados, está marcada por el constante cambio en el ámbito de la empresa. Las empresas constantemente se están transformando en respuestas a modificaciones en el entorno, cambios en la regulación, nuevas tecnologías y preferencias de los consumidores. Como consecuencia de este rápido proceso de transformación, las teorías que se generan para explicar el comportamiento en los mercados y los resultados empresariales fácilmente pierden su vigencia, por lo que continuamente deben ser verificadas ante la realidad $y$, en su caso ajustadas o sustituidas, con el fin de incorporar las anomalías detectadas (Kuhn, 2006).

La investigación con estudio de casos es particularmente apropiada para ciertos tipos de problemas (Bonoma, 1985): donde la investigación y la teoría se hallan en sus fases preliminares y para problemas prácticos delicados donde las expe- riencias de los participantes son importantes y el contexto de la situación es fundamental. Así, los estudios de casos pueden ser empleados para documentar las experiencias que se llevan a cabo en las empresas y permiten obtener explicaciones ricas y convincentes de los resultados basados en los datos y en la documentación existente, para de esta manera lograr lo que se define como rigor, que es el equivalente a evidencias convincentes y bien fundamentadas (Keen, 1991).

Existen tres razones por las que la investigación mediante estudios de casos es un modo de investigación viable en Economía de la Empresa (Cepeda, 2006). Primero, porque el investigador puede estudiar el fenómeno objetivo o la empresa o en su estado natural, aprender de la situación, y generar teorías a partir de todo lo encontrado. Segundo, el método del caso le permite al investigador responder al cómo y al porqué, esto es, comprender la naturaleza y complejidad de los procesos que toman lugar. Por último, el estudio de casos es una manera apropiada de investigar en un tema en el cual se han desarrollado pocos o ningún estudio anteriormente.

\section{JUSTIFICACIÓN ESTUDIO DE CASOS}

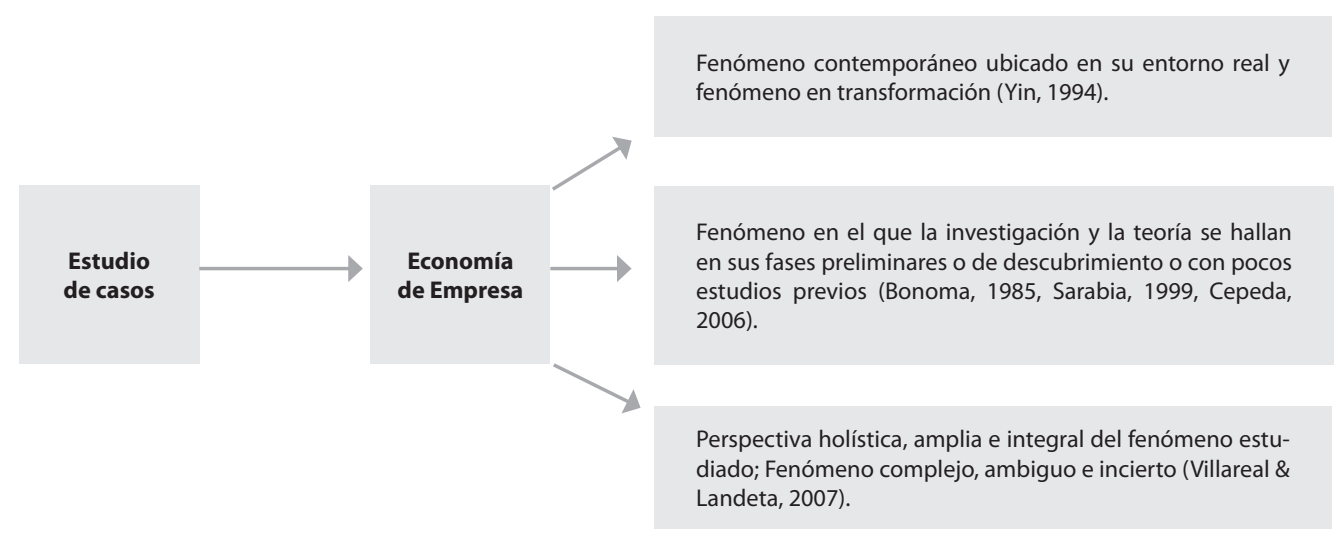

Fuente: elaboración propia con base en la literatura. 


\section{Cómo desarrollar el estudio de casos}

\section{La selección de los casos objeto de estudio y la unidad de análisis}

En todo estudio de casos debe existir una frontera espacial y temporal (VanWynsberghe \& Khan, 2007). La selección de los casos es de suma importancia, ya que en esta etapa se procede a la definición de la unidad de análisis o de observación que es lo que constituye el caso a estudiar. Según Sierra (1994), "las unidades de observación son las realidades que se pretenden observar. Como tales constituyen en la investigación el objeto global del estudio y de ellas se obtienen los datos empíricos para corroborar las proposiciones o hipótesis con la realidad" (p. 96). En el estudio de casos, la definición de la unidad de análisis ayuda a definir las fronteras de la teoría. La clara selección de la población corrobora que la unidad de análisis es consistente con los límites de la teoría que se intenta probar (Dubé \& Paré, 2003).

Merriam (1988), recomienda el estudio de casos cuando el centro de la investigación sea un sistema integrado, una persona, un proceso, una institución o un grupo social. Por su parte, Yin (1994) señala que las unidades de análisis permiten definir qué es el caso. Cuando el estudio de caso se realiza sobre un objeto concreto, la unidad de análisis está clara porque es el propio objeto investigado. En cambio, en el estudio de casos sobre fenómenos o acontecimientos muy complejos, se hace necesario considerar una o varias unidades de análisis que permitan concretar la investigación. En definitiva, las unidades de análisis permiten definir los límites del caso logrando así diferenciarlos de su contexto y orientando la elaboración de los resultados. Es así como la unidad de análisis, que es lo que constituye el caso a estudiar, puede ser una entidad, un individuo o un acontecimiento.
Otra definición importante es la que ofrece Benbasat, Goldstein y Mead (1987) que conceptualizan el estudio de casos como "aquella investigación que usa múltiples métodos y herramientas para la recogida de datos por un observador directo de varias entidades, en una escena úni$\mathrm{ca}$, considerando aspectos temporales y contextuales del fenómeno bajo estudio, pero sin experimentación ni manipulación" (p. 370). Para Langley y Royer (2006), cuando se habla de entidades, se está hablando del foco específico de estudio y éste puede ser una persona, un grupo, una organización, una relación, un acontecimiento, un proceso, un problema o cualquier otra entidad específica.

Cuando se realiza una investigación con estudios de casos, la unidad de análisis debe ayudar a delimitar, en lo posible, las fronteras del estudio; si bien es cierto resulta a veces muy difícil separar un determinando fenómeno del contexto en el que éste se produce. La unidad de análisis está relacionada con el problema fundamental de decidir el tipo de caso que es. Como guía general, la definición de la unidad de análisis, y con ello del tipo de caso, se relaciona con la forma en que ha sido definida la cuestión de investigación inicial (Rialp, 1998; VanWynsberghe y Khan, 2007).

Tras definir la unidad de análisis, la selección del caso o casos a estudiar se basará en un muestreo teórico, no estadístico, tratando de escoger aquellos casos que ofrezcan una mayor oportunidad de aprendizaje (Stake, 1994) y que permitan una generalización analítica de los resultados. Se trata de determinar el núcleo sobre el cuál estudiar las cuestiones que se plantean, que se van a analizar: una empresa, un grupo de empresas, relaciones entre éstas o un fenómeno. Es importante mencionar que muchas veces la propia investigación lleva a la unidad de análisis y los investigadores definen las unidades de análisis conforme transcurra el avance de la investigación (Ragin \& Becker, 1992; Easton, 1994; Yin, 1994; VanWynsberghe \& Khan, 2007). 
Cuando estén definidas las unidades de análisis el investigador se centra sólo en los aspectos del fenómeno estudiado que concierne a las proposiciones que están examinando. Esto no implica que se omita el análisis del contexto, pero focaliza la atención y permite disminuir el tiempo destinado al trabajo de campo. Lo recomendado es que se establezcan las menor cantidad de unidades de análisis posibles (Fong, 2008).

Según Yin (1994), un estudio de casos puede involucrar más de una unidad de análisis si el interés de la investigación está puesto en otra u otras subunidades (por ejemplo, un estudio sobre organización puede centrarse en ciertos roles o determinadas formas de interacción) incorporadas en la entidad de referencia. En esta situación, se hablará de estudio de casos incorporados o sentido incrustado o encajado. En cambio, si el estudio sólo implica la naturaleza global o de conjunto de la organización, comunidad o proceso que interesa, se está en un diseño holístico. El criterio principal para optar por uno u otro diseño es el grado de focalización que se requiere alcanzar dentro del caso en estudio. Es decir, las diferencias entre estos diseños dependen de los fenómenos por estudiar y de los intereses de conocimiento que sobre ellos prima: más holístico o más focalizado. En resumen, si se sintetizan estas aportaciones, los interrogantes “cómo?" y“ ¿por qué?" permiten concretar el problema inicial de una investigación de estudio de caso donde será imprescindible identificar un sistema integrado que constituirá el fenómeno objeto de estudio.

De esta manera, Yin (1994) resume que los estudios de casos pueden ser simples o múltiples, dependiendo del número de casos que se vaya a estudiar y de los diferentes niveles o unidades de análisis. Así identifica:

- Un único caso, el cual no se puede descomponer en subunidades o unidad de análisis.
- Un único caso el cual se descompone en distintas subunidades.

- Casos múltiples con unidad principal de análisis que no se pueden descomponer en subunidades.

- Casos múltiples que se descomponen en distintas subunidades.

En la investigación, la selección de los casos que conforman el estudio de investigación se realiza por razones teóricas más que estadísticas (Yin, 1994). Debido a que el número de casos a estudiar suele ser limitado, existen, en la teoría, unas reglas básicas para la elección de los casos. Se considera que si el fenómeno a estudiar está contenido en un caso o relativamente en pocos casos, es necesario elegir los casos donde el progreso sea transparentemente observable, tales como aquellos en los que existan situaciones extremas, incidentes críticos y dramas sociales (Eisenhardt, 1989; Pettigrew, 1990; Meredith, 1998).

\section{Recolección de la información en el estudio de casos}

Después de definir los casos a estudiar, las unidades de análisis y los procedimientos para recopilar la información, se hace necesario proceder a la obtención de la información.

Según indican Hernández, Fernández y Baptista (2003), en una investigación cualitativa, la recolección de datos resulta fundamental y su propósito no es medir variables para llevar a cabo inferencias y análisis estadísticos. Lo que se busca es obtener información de sujetos, comunidades, contextos, variables o situaciones en profundidad. El investigador cualitativo utiliza una postura reflexiva y trata, lo mejor posible, de minimizar sus creencias, fundamentos o experiencias de vida asociados con el tema de estudio. Se trata de que éstos no interfieran en la recolección de 
datos y de obtener los datos de los sujetos, tal y como ellos lo revelan.

Los datos cualitativos consisten en la descripción profunda y completa de eventos, situaciones, imágenes mentales, interacciones, percepciones, experiencias, actitudes, creencias, emociones, pensamientos y conductas reservadas de las personas, ya sea de manera individual, grupal o colectiva. Los datos se recolectan con la finalidad de analizarlos para comprenderlos y responder así a preguntas de investigación o generar conocimiento. La recolección de datos ocurre completamente en los ambientes naturales y cotidianos de los sujetos. Esta clase de datos es muy útil para comprender los motivos subyacentes, los significados y las razones internas del comportamiento humano. (Hernández, Fernández \& Baptista, 2003).

Para evitar inconvenientes en la investigación, es necesario, en la mayoría de los estudios de casos, utilizar combinación de técnicas para obtener la información, tales como cuestionarios, revisión de documentos y colaboración de personas expertas en el sector estudiado (Dawson, 1997; Snow \& Thomas, 1994; Fox-Wolfgramm, 1997). Por tanto, las fuentes de información que se pueden utilizar en un estudio de casos son múltiples. Asimismo, no se debe considerar que ninguna fuente tenga una ventaja absoluta sobre las demás, más bien, las distintas fuentes son altamente complementarias, por lo que para realizar un buen estudio de casos se utilizan todas las que sean posibles, lo que permite a la vez contrastar diferentes tipos de datos (Eisenhardt, 1989; Yin, 1994).

\section{Evaluación de los estudios de casos: validez y fiabilidad de la investigación}

Sutton (1997) señala que, para que una investigación sea considerada valiosa y aceptable, tiene que ser rigurosa, por lo que debe ser fiable, válida y generalizable. Por dichas razones, esta metodología ha tenido críticas de algunos autores, las cuales se relacionan con el papel del investigador -sesgo del investigador-, el cual especifica el fenómeno a estudiar, elige el marco teórico, pondera la relevancia de las distintas fuentes y analiza la conexión causal entre hechos. Esta crítica se fundamenta, según los autores, en que éstos no son objetivos, porque no se sabe en qué medida el estudio se corresponde con los hechos o refleja la percepción subjetiva del investigador, y además son poco fiables, ya que si otra persona vuelve a estudiar el mismo caso, probablemente elaboraría un estudio diferente. Sin embargo, Bonache (1999) señala que los autores que defienden esta metodología alegan que los métodos estadísticos de ninguna manera controlan el sesgo del investigador, ya que éste impone el marco teórico, imposibilitando que aparezcan respuestas imprevisibles.

Van de Ven (1992) por su parte recomienda tener sumo cuidado con el marco de referencia temporal cuando se realiza el diseño de la investigación. Adicionalmente, el investigador se enfrenta a problemas relacionados con las limitaciones cognitivas y de percepción del observador, ya que el investigador puede influir en el sistema que está observando y debe asegurarse de interpretar correctamente lo que está observando; el investigador, en la medida que usa a los participantes como informantes clave, se enfrenta a los problemas de identificar a los mejores informantes y asegurarse de que comprenden correctamente la investigación y que proporcionan información verídica.

Los aspectos sobre la validez y la fiabilidad de una investigación realizada con estudio de casos, siempre han supuesto que los investigadores que utilicen dicha metodología realicen un importante esfuerzo para fijar criterios y métodos tan rigurosos como los seguidos por las investigaciones de corte cuantitativa (Numagami, 1998). 
Para Chetty (1996), es necesario e importante analizar las investigaciones con el objetivo de establecer los criterios externos que puedan emplearse en determinar la utilidad de las diversas maneras en que se estudiará el asunto en cuestión; por lo que deben utilizarse dos criterios para su evaluación: la validez interna y la validez externa. Yin (1994) señala que, para establecer la calidad de cualquier investigación socio-empírica, se puede considerar la existencia de cuatro aspectos que son utilizados comúnmente y que se muestran relevantes para evaluar la calidad de la investigación realizada mediante estudios de casos. El autor hace referencia, en este sentido, a los siguientes criterios:

- Validez del modelo o de los constructos

- Validez interna

- Validez externa

- Fiabilidad

Este conjunto de indicadores son los que más habitualmente se sugieren y documentan en la literatura y constituyen el mejor modo de mejorar y valorar la validez y fiabilidad de los estudios de casos (Riege, 2003; Gibbert et al., 2008).

\section{La validez del modelo}

Yin (1994) señala que la validez del modelo tiene varios aspectos que son fundamentales: deben abarcarse todos los conceptos que deben analizarse, las medidas operativas que los caractericen deben ser verdaderos indicadores del fenómeno y debe elegirse una forma eficiente que realmente sea un evaluador de todo que se va a investigar. Yin (1994) señala que para lograr mayor validez, se deben utilizar varias fuentes de evidencia, establecer una cadena de evidencia y exponer a revisión de expertos los resultados preliminares de la investigación:
- La utilización de múltiples fuentes de evidencia es de suma importancia, de forma que se aliente la convergencia de las líneas de indagación y que no se cuestione el resultado de lo medido, es una táctica relevante en la fase de recopilación de datos.

- Establecer una cadena de evidencias es necesario para poner de manifiesto el razonamiento del investigador y permitir al lector de un estudio de casos obtener conclusiones al analizar las evidencias. Para ello, es recomendable que el informe final del estudio de casos presente suficientes citas y referencias a la base de datos del estudio donde se incluye toda la información recogida por los diversos procedimientos -entrevistas, cuestionarios, revisión documental, análisis de archivos entre otros-.

- La redacción de un borrador del informe del estudio de casos, para que sea revisado por expertos en la materia que hayan participado en la investigación. De esta manera, se tiene la oportunidad de corregir errores y detectar aquella información comercialmente sensible que no puede ser incluida (Pettigrew, 1990).

\section{La validez interna}

La validez interna de un estudio de casos hace énfasis en el grado en que el estudio se convierte en una investigación objetiva, ya que refleja y explica la verdadera situación analizada (Bonache, 1999).

Para Yin (1989), la validez interna es el grado en el cual se pueden establecer relaciones causales, donde ciertas condiciones demuestran conducir a otras. En su opinión, para garantizar la validez interna, las tácticas aconsejadas están relacionadas con la utilización de los siguientes cuatro aspectos: la triangulación, el ajuste a un patrón, 
la construcción de explicaciones y el análisis de series temporales.

1) La triangulación, tecnicismo procedente de la navegación, consiste en la utilización de al menos tres puntos de referencia para la localización de un objeto, por lo que en la investigación se asocia con el uso de múltiples e independientes medidas (Chiva, 2001). Al recurrir a la triangulación metodológica, la información obtenida de los cuestionarios se completa con la observación directa y la revisión de documentos. Los documentos son de muy diversa naturaleza -informes, entrevistas, artículos, noticias, páginas web donde se puede observar todo tipo de información importante para el estudio de investigación-. La revisión de la literatura también sirve para elegir las cuestiones que son objeto de estudio.

Patton (1987) recomienda la triangulación de la información de tres maneras: triangulación de fuentes de datos, triangulación de las técnicas de recopilación de la información y triangulación del investigador. Por otra parte Easterby, Thorpe y Lowe (1991) consideran cuatro grupos de triangulación importantes para la investigación:

a) Teórica, que consiste en utilizar modelos teóricos de una disciplina para explicar situaciones en otras.

b) De datos, que se refiere a la utilización de datos que viene de distintas fuentes o en diferentes momentos del tiempo.

c) De investigadores, que consiste en comparar datos obtenidos por otros investigadores sobre el mismo fenómeno.

d) Metodológica, que se basa en el uso de diferentes métodos o técnicas a la hora de recoger lo datos.
2) La lógica de ajustar o emparejar patrones tiene que ver con la comparación de un patrón obtenido empíricamente con otros basados en predicciones teóricas, ya que si los resultados coinciden, su validez interna es mayor. De ahí la importancia de la revisión y análisis de la literatura relacionada con el fenómeno a estudiar.

3) La construcción de una explicación por su parte, tiene que ver con el aporte de aclaraciones detalladas y razonadas de todas las afirmaciones y datos sobre el estudio realizado. El objetivo es analizar los datos del estudio, estableciendo un conjunto de vínculos causales sobre el mismo, que en muchos estudios pueden ser difíciles y complejos de medir de una manera precisa.

4) El análisis de series temporales consiste en estudiar, explicando el cómo y el porqué de un determinado fenómeno a lo largo del tiempo (Easterby, Thorpe y Lowe, 1991).

\section{La validez externa}

La validez externa establece el dominio en el cual pueden generalizarse los hallazgos del estudio (Kidder \& Judd, 1986). En otras palabras, la validez externa hace alusión a la capacidad de generalización de las conclusiones de la investigación estudio de casos. En este mismo sentido, se considera que la validación externa es el punto más débil de la metodología del caso; es así como algunos autores afirman que el estudio de casos tiene una base muy débil para la generalización de sus resultados, lo que contrasta con otras estrategias de investigación que contienen indicadores estadísticos. Sin embargo, como ya fue mencionado, esta analogía a las muestras y a los universos no es correcta cuando se habla de estudios de casos, dado que estas investigaciones se basan en la generalización analítica de un conjunto particular de resultados a una teoría más amplia (Yin, 1989). 
De hecho, la generalización estadística, aunque sea el modo más utilizado en los trabajos empíricos de organización de empresas, no es el único tipo existente de generalización. El conocimiento científico se caracteriza por la búsqueda de generalidades teóricas, constituyendo la lógica de la réplica, la vía esencial para lograrlas, de forma similar a la utilizada en los estudios de casos múltiples. Además, esta lógica no se basa en la inducción estadística, sino en la analítica y trata de ver lo general en lo particular. Es decir, en lugar de generalizar los resultados a una población, se intenta descubrir en casos concretos las causas o condiciones generales que permitan explicar y predecir un fenómeno (Bonache, 1999). Por otra parte, se adquiere validez externa en una investigación al analizar las experiencias de los casos bajo estudio, recurriendo a las citas textuales de las opiniones y respuestas otorgadas por los entrevistados, método recurrido ampliamente para lazar las perspectivas de los participantes (Dubé \& Paré, 2003). En este mismo sentido, Yin (1989) señala que la lógica que subyace en la selección de casos es la lógica de la réplica, la cual plantea que cada caso debe ser seleccionado con mucho cuidado, de forma que cumpla dos requisitos que son fundamentales: réplica literal o sea que prediga resultados similares a otro caso y la réplica teórica que es cuando genera resultados opuestos a los otros casos pero por razones predecibles. La lógica de la réplica otorga al estudio la importante validez externa, ya que permite esperar que en casos con condiciones similares se puedan realizar estudios que den resultados parecidos o iguales.

\section{La fiabilidad}

Yin (1994) establece la fiabilidad como el criterio para demostrar que las operaciones de un estudio, los procedimientos de recopilación de datos, pueden repetirse con los mismos resultados. Así, la fiabilidad se refiere al grado de seguridad o consistencia. Un estudio es más fiable cuanto mayor es la consistencia de sus mediciones (Yacuzzi, 2005), es decir, que si otra persona repitiese el estudio de caso obtendría resultados similares. De esta manera, lo que se pretende es minimizar los errores y los sesgos en el estudio realizado, destacando la importancia de determinar un protocolo del caso con la especificación de todos los pasos que han sido seguidos, así como una base de datos que tenga toda la información recopilada para responder, de esta manera, con detalles ante un hipotético auditor que solicite su justificación (Bonache, 1999).

A continuación, en la tabla 2, se presenta el resumen de la validez y fiabilidad del estudio de casos.

TABLA 2

\section{RESUMEN VALIDEZ Y FIABILIDAD DEL ESTUDIO DE CASOS}

\begin{tabular}{ll}
\hline & \multicolumn{1}{c}{ VALIDEZ EN EL ESTUDIO DE CASOS } \\
\hline $\begin{array}{l}\text { VALIDEZ } \\
\text { INTERNA }\end{array}$ & $\begin{array}{l}\text { Considera el establecimiento de relaciones causa-efecto. En los estudios de casos consiste en presentar el fenómeno de una for- } \\
\text { ma creíble. Los investigadores no solo manifiestan las similitudes y diferencias de las experiencias y creencias de los participantes } \\
\text { sino que además intentan identificar qué aspectos son significativos en el fenómeno a estudiar y qué mecanismos lo producen. }\end{array}$ \\
\hline $\begin{array}{l}\text { VALIDEZ } \\
\text { CONSTRUCTO }\end{array}$ & $\begin{array}{l}\text { Facilita el desarrollo de un conjunto de medidas mínimamente operativas y trata de evitar los juicios subjetivos de los investiga- } \\
\text { d) Utilizar múltiples fuentes de evidencias; b) Usar una cadena de evidencias y/o razonamientos; } \text { c) Hacer que terceras personas, } \\
\text { con conocimiento del fenómeno y/o situación a estudiar, revisen los resultados del caso. }\end{array}$ \\
\hline $\begin{array}{l}\text { VALIDEZ } \\
\text { EXTERNA }\end{array}$ & $\begin{array}{l}\text { Se refiere a si los resultados del caso son generalizables más allá de la situación concreta que se estudia. El tipo de generalización } \\
\text { que se utiliza en los estudios de casos es la denominada "generalización analítica" en la que una teoría previamente desarrollada } \\
\text { puede ser utilizada para contrastar los resultados empíricos del caso. }\end{array}$ \\
\hline FIABILIDAD & $\begin{array}{l}\text { Un estudio de casos es fiable si podemos obtener resultados similares siguiendo el mismo protocolo que los anteriores investiga- } \\
\text { dores han desarrollado. }\end{array}$ \\
\hline \hline
\end{tabular}

Fuente: Adaptado de Cepeda, 2006 
Para finalizar se puede hacer un resumen de las diferentes etapas o fases necesarias para la realización de un estudio de casos, las cuales se presentan en la Figura 2.

FIGURA 2

PROCEDIMIENTO METODOLÓGICO DE LA INVESTIGACIÓN UTILIZADO PARA UN ESTUDIO DE CASOS

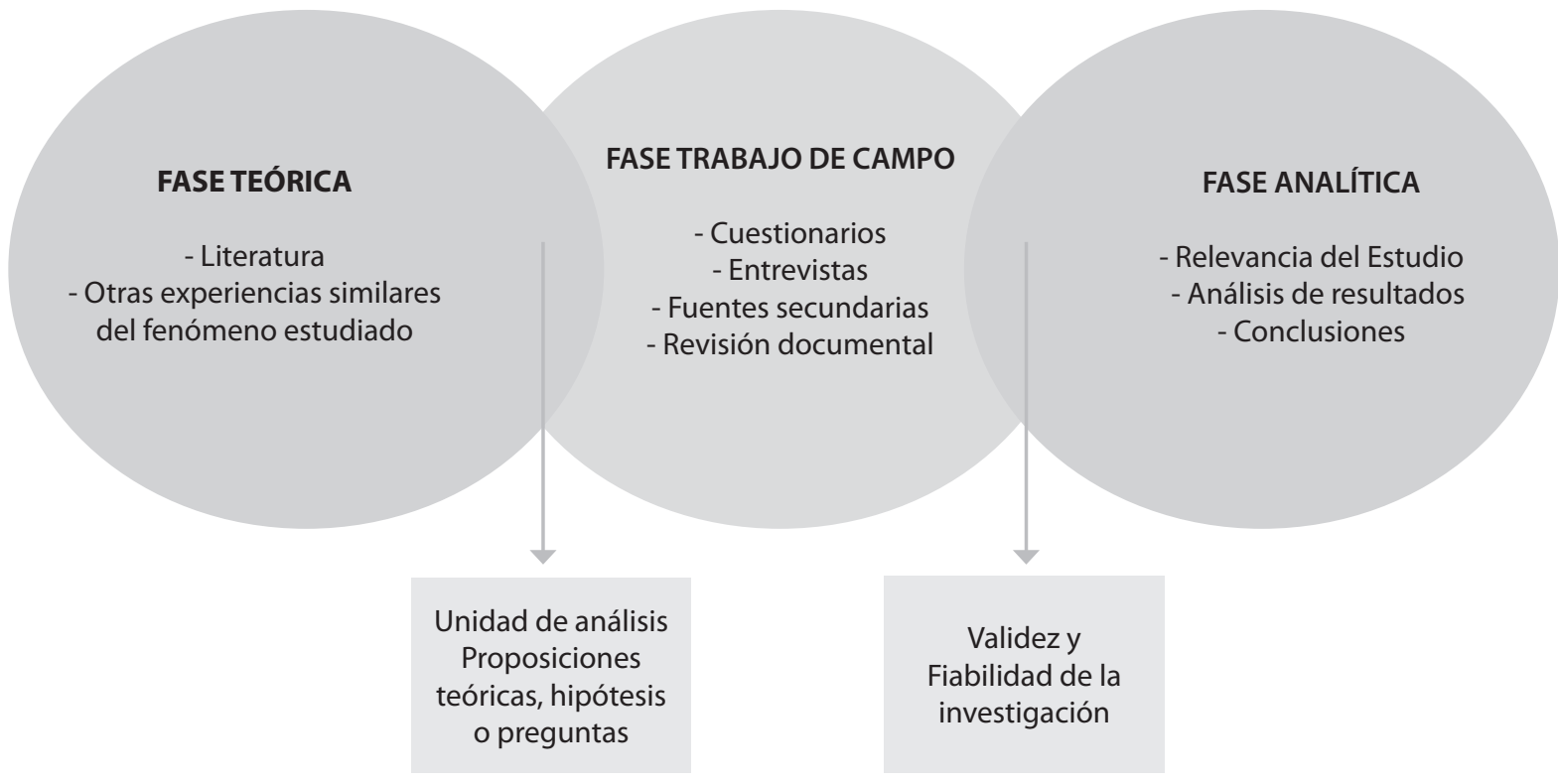

Fuente: elaboración propia basado en la literatura.

\section{CONCLUSIONES}

Ha quedado de manifiesto que existen dos enfoques principales de investigación: el cuantitativo y el cualitativo, y que en el presente pueden formar parte de un mismo estudio. El estudio de casos como método cualitativo le brinda, a las empresas u organizaciones que lo utilizan, importantes datos que no ofrece el método cuantitativo, de ahí el importante auge que el estudio de casos tiene en el presente.

En la realización del estudio de casos debe existir una frontera espacial y temporal y debe definirse una o varias unidades de análisis, que en realidad es lo que constituye el caso a estudiar. Si no se define bien la unidad de análisis, no se están estableciendo bien los límites de la investigación y los resultados no estarán bien orientados. Ya establecida las unidades de análisis se establecen hipótesis, proposiciones teóricas o preguntas de investigación que guían al investigador, focalizando de esta manera la atención en lo que se desea investigar y destinando así el tiempo efectivo en el trabajo de campo.

La selección de los casos que componen la investigación es de suma importancia y ésta se realiza más por razones teóricas que estadísticas 
y puede ser un único caso o múltiples casos con una o varias unidades de análisis.

Un buen estudio de casos debe tener validez interna, reflejando que la investigación es objetiva, analizando y explicando la verdadera situación estudiada. Asimismo, debe darse la investigación con validez externa que brinda la capacidad de que sus conclusiones puedan ser generalizadas analíticamente a teorías más amplias. Si se pretende que la investigación sea valiosa, debe ser fiable, válida y rigurosa por lo que el investigador debe realizar un gran esfuerzo por establecer criterios rigurosos que le den la confianza de estar realizando un buen estudio de casos. Para cumplir con los parámetros de validez es necesario utilizar varias fuentes de información y diversos procedimientos como entrevistas, cuestionarios, revisión de documentos, análisis de archivos, entre otros, y someter luego a revisión de expertos los resultados provisionales que la investigación arrojó.

Queda expresado entonces, el auge del uso de técnicas cualitativas como el estudio de casos en diferentes áreas de la administración de empresas. Las organizaciones se han convertido en fenómenos realmente complejos que requieren de investigaciones que exploren y generen teoría para las organizaciones y para la sociedad. El estudio de casos es muy apropiado en los fenómenos nuevos, en donde la teoría se encuentre en sus fases preliminares, y sirve para documentar las experiencias que se dan en las organizaciones.

En el presente, los gerentes de las empresas requieren de información de primera mano producto de análisis e investigaciones profundas que generen un proceso eficiente de toma de decisiones y el estudio de casos se convierte en una herramienta de apoyo a dicho proceso.

\section{REFERENCIAS}

Benbasat, I., Goldstein, D. \& Mead, M. (1987). The case research Strategy in studies of information systems. MIS Quarterly, 3 (11), 369-386.

Bonache, J. (1999). El estudio de casos como estrategia de construcción teórica: características, críticas y defensas. Cuadernos de Economía y Dirección de la Empresa, 3, 123-140.

Bonoma, T. (1985). Case Research in Marketing: Opportunities, Problems, and a Process. Journal of Marketing Research, 22 (2), 199-208.

Bryman, A. (1984). The debate about quantitative and qualitative research: a question of method or epistemology. The British Journal of Sociology, 35, 75-92.

Burgelman, R. (1994). Fading memories: a process theory of strategic business exit in dynamic environments. Administrative Science Quarterly, 39, 24-56.

Carlson, E., Engebretson, J. \& Chamberlain, R. (2005). The Evolution of Theory: A Case Study. International Journal of Qualitative Methods, 4 (3), 20-36.

Cepeda, C. (2006). La calidad en los métodos de investigación cualitativa: principios de aplicación práctica para estudios de casos. Cuadernos de Economía y Dirección de la Empresa. (ACEDE) n. 29. 057-082.

Chetty, S. (1996). The case study method for research in small and medium sized firms. International Small Business Journal, 15, 73-85.

Chiva, R. (2001). El estudio de casos explicativo. Una reflexión. Revista de Economía y Empresa, 41, 119-132.

Consuelo, M. (2005). La investigación cualitativa en marketing: el camino hacia una percepción social del mercado. Revista Colombiana de Marketing. 4 (6), 70.

Coser, L. \& Hunt, CH. (1994). Sociological Theory. 5a. edición, New York: Waveland Press.

Creswell, J. (1997). Qualitative inquiri and research designs: Choosing harmony among five traditions. Thousand Oaks, CA: Sage Publications.

Dachler, H. (1997). Does the distinction between qualitative and quantitative methods make sense. Organization Studies, 18(4), 709-724.

Dawson, P. (1997). In at the deep end: conducting processual research on organisational change. Scandinavian Journal of Management, 13, 389-405. 
Dess, G. \& Davis, P. (1984). Porter-1980-generic strategies as determinants of strategic group membership and organizational perfomance. Academy of Management Journal, 27, 467-488.

Dubé, L. \& Paré, G. (2003). Rigor in information systems positivist case research: Current practices, trends, and recommendations. MIS Quarterly, 27 (4), 597-635.

Dyer \& Wilkins (1991). The Case Study Method for Research in Small-and Medium-Sized Firms. Chetty International Small Business Journal. 1996; 15, 73-85.

Easterby, M., Thorpe, R. \& Lowe, A. (1991). Management research: an introduction. Londres, Sage Publications.

Easton, G. (1994). Methodology and industrial networks, in Wilson, D.T., Moller, K. (Eds), Relationships and Networks: Theory and Application, PWS, Kent.

Eisenhardt, K. (1989). Building theories from case study research. Academy of Management Review, 14, 532-550.

Eisenhardt, K. (1991). Better stories and better constructs: the case for rigor and comparative logic. Academy of Management Review, 16 (3), 620-627.

Eisenhardt, K \& Graebner, M. (2007). Theory building from cases: opportunities and challenges. Academy of Management Journal, 50 (1), 25-32.

Fong, C. (2002). El estudio de casos en la preparación de tesis de posgrado en el ámbito de la PYME. XXII Congreso anual de AEDEM, Salamanca, España.

Fox-Wolfgramm, S. (1997). Towards developing a methodology for doing qualitative research: the dynamic-comparative case study method. Scandinavian Journal of Management, 13, 439-455.

Ghauri, P., Gronhaug, K. \& Kristianslund, I. (1995). Research methods in business studies: a practical guide. Prentice Hall, Nueva York.

Gibbert, M.; Ruigrok, W.; Wicki, B. (2008). Research notes and comentaries. Waht as a passes as a rigorous case study? Strategic Management Journal, 29, 1465-1474.

Grinell, R. (1997). Social work research \& evaluation: Quantitative and qualitative approaches. E.E. Peacock Publishers, 5.ed. Illinois.

Grunow (1995). The research design in Organization Studies: problems and prospects. Organization Science, 6, 93-103.
Guba, E. \& Lincoln, Y. (1994). Competing Paradigms in Qualitative Research. En: Denzin NK Lincoln. Handbook of Qualitative Research. Thousand Oaks: SAGE; 105-117.

Gummesson, E. (2000). Qualitative methods in management research. Sage Publications, London.

Hamel, J. (1992). The Case method in Sociology, Introduction: New Theoretical and Methodological Issues. Current Sociology, 40 (1), 1-7.

Hamel, J. , Dufour, S. \& Fortín, D. (1993). Case Study Methods Sage Publications, Newbury Park, California.

Hartley, J. (1994). Case studies in organizational research, en CASELL, C. y SYMON, G. (Eds.). Qualitative methods in organizational research. Londres, Sage Publications, 208-229.

Hernández, R., Fernández, C., Baptista, P. (2003). Metodología de la Investigación. Tercera edición. Editorial Mc Graw Hill. México.

Keen, P. (1991). Relevance and rigor in information systems research: improving quality, confidence, cohesion and impact. H.E. Nissen, H.K. Klein y R. Hirschheim (eds.), Information System Research: Contemporary approaches and emergent traditions, Elsevier, 27-49.

Kidder, L. \& Judd, C. (1986). Research Methods in Social Relations. Quinta edición, CBS College Publishing, New York.

Kuhn, T. (2006). La estructura de las revoluciones científicas. Fondo de Cultura Económica. México D.F.

Langley, A. \& Royer, I. (2006); Perspectivas on Doing Case Study Research in Organizations. Management, 9 (3), 73-86.

Larsson, R. (1993). Case Survey Methodology: Qualitative Analysis of Patterns across Case Studies. Academy of Management Journal, 36, 1515-1546.

Leonard, D. \& Mcadam, R. (2001). Grounded theory methodology and practitioner reflexivity in TQM research. International Journal of Quality \& Reliability Management, 18, 180-194.

Meredith, J. (1998). Building operations management theory through case and field research. Journal of Operations Management, 16, 441-454.

Merrian, S. (1998). Qualitative Research and Case Study Applications in Education. 2. ed. Jossey-Bas Inc. 
Miles, M. \& Huberman, A. (1994). Qualitative Data Análisis. 2 ed., Sage Publications.

Mintzberg, H. (1979). An Emerging Strategy of Direct. Research, Administrative Science Quarterly, 24 (4), 582589.

Mintzberg, H. (1990). Strategy formation: Schools of thought. (Ed.). Perspectives on Strategic Management. Nueva York, Harper Business:105-235.

Morgan, G. \& Smiricich, L. (1980). The case for qualitative research. Academy of Management Review, 5 (4), 491500.

Neuman, W. (1991). Social research methods: qualitative and quantitativ approaches. Boston. Allyn and Bacon.

Numagami, T. (1998). The infeasibility of invariant laws in management studies: a reflective dialogue in defense of case studies. Organization Science, 9, 3-15.

Orozco, G. (1996). La investigación en comunicación desde la perspectiva cualitativa. U.N.L.P., La Plata, Argentina.

Otley, D. \& Berry, A. (1994). Case Study research in management accounting and control. Management Accounting Research, 5, 45-65.

Patton, M. (1987). How to use Qualitative methods en Evaluation. Newbury Park, C.A, Sage.

Patton, M. (1990). Qualitative evaluation and research methods. (2nd ed.). Newbury Park, CA. Sage

Pettigrew, A. (1990). Longitudinal field research on change: theory and practice. Organization Science, 1, 267-291.

Ragin, C. \& Becker, H. (1992). What is a Case. Exploring the Foundations of Social Inquiry. Cambridge: University Press.

Rialp, A. (1998): El método del caso como técnica de investigación y su aplicación a la función directiva. Ponencia presentada en el IV Taller de metodología ACEDE, 2325 de abril, Arnedillo, La Rioja.

Riege, A. (2003): Validity and reliability test in case study research: a literatura with hands on applications for each research phase. Qualitative Research: An International Journal, 6 (2), 75-86.

Robinson, R. \& Pearce, J. (1988): Planned patterns of strategic behavior and theirrelationship to business unit performance. Strategic Management Journal, 9, 43-60.
Sarabia, F. (1999): Metodología para la investigación en Marketing y dirección de empresas. Editorial Pirámide. Madrid- España.

Sierra, R. (1994): Técnicas de Investigación Social, Ed. Paraninfo, Madrid. España.

Siggelkow, N. (2007): Persuasion with case studies. Academy of Management Journal, vol. 50(1), 20-24.

Silverman, (1993): Interpreting Qualitative Data: Methods for analyzing talk, text and interaction. Sage Publications, London.

Smith, A. \& Zeithaml, C. (1999): The Intervening Hand: Contemporary Intenational Expansion Process of the Regional Bell Operating Companies. Journal of Management Inquiry, 8, 34-64.

Snow, C. \&Thomas, J. (1994): Field research methods in strategic management: contributions to theory building and testing. Journal of Management Studies, 31, 457480.

Sosa, S. (2003): La Génesis y el Desarrollo del Cambio Estratégico: Un Enfoque Dinámico basado en el Momentum Organizativo. Tesis Doctoral, Universidad de las Palmas de Gran Canaria.

Stake, R. (1995): The art of case study research. London, Sage Publications.

Stoecker, R. (1991): Evaluating and rethinking the case study. Social Review, 0038-0261, 88-112.

Stopford, J. \& Baden-Fuller, C. (1994): Creating corporate entrepreneurship. Strategic Management Journal, 15, 521-536.

Strauss, A. \& Corbin, J. (1990): Basics of qualitative research : grounded theory procedures and techniques. Newbury Park London: Sage.

Sutton, R. (1997): The virtues of closet qualitative research. Organization Science, 8, n. 1, 97-106.

Taylos, S. \& Bogdan, R. (1996): Introducción a los métodos cualitativos de investigación. Editorial Paidos, Barcelona.

Van de Ven, A. (1992): Suggestions for studying strategy process: A research note. Strategic Management Journal, 13, 169-188.

VanWynsberghe, R., \& Khan, S. (2007): Redefining case study. International Journal of Qualitative Methods, 6 (2), 80-94. 
Villareal, O. \& Landeta, J. (2007): El estudio de Casos como metodología de investigación científica en Economía de la Empresa y dirección Estratégica. Universidad del País Vasco.

Volverda, H. (1997): Building flexible organizations for fastmoving markets. Long Range Planning, 30, 169-183.

Voss, CH., Tsikriktsis, N. \& Frohlich, M. (2002): Case research in operations management. International Journal of Operations and Production Management, 22 (2), 195-219.

Vouzas, F. \& Kufidu, S. (2003). The context and content of strategic human resources management in selected greek organizations: a case study aprproach, en Universidad de Cádiz (Ed). La gestión de Recursos Humanos en la Nueva Economía. Universidad de Cádiz.

Weber, R. (2004): The Rethoric of Positivism versus Interpretivism: A personal view. MIS Quarterly, 28 (1), 3-12.

Weick, K. (2007): The generative properties of richness. Academy of Management Journal, 50 (1), 14-19.

Wright, L. (1996): Qualitative International Management Research. Eds: Handbo for International Management Research, Blackwell, 63-81.

Yacuzzi, E. (2005). El Estudio de Caso como metodología de investigación: Teoría, mecanismos causales, validación. Universidad del CEMA, Buenos Aires, Argentina, 1-37.

Yan, A. \& Gray, B. (1994): Bargaining Power, Management Control, and Perfomance in United States-China Joint Venture: A Comparative Case Study. Academy of Management Journal, 37 (6), 1478-1517.

Yin, R. (1981): The case study crisis: Some answers. Administrative Science Quarterly, 6 (1), 58-65.

Yin, R. (1989): Case Study Research: Design and Methods. Ed. Sage, Newbury Park, CA.

Yin, R. (1994): Case Study Research: Design and Methods. Sage Publications, Thousand Oaks, CA.

Recibido: 23-04-2010

Aceptado: 27-06-2010 